\title{
El rasgo testimonial en la semántica del pasado búlgaro
} \section{Alfons Olivares Niqui}

Universitat de Barcelona

Departament de Lingüística General

Gran Via de les Corts Catalanes 585

08007 Barcelona

Teléfono +34934035583

Fax +34934035596

RESUMEN El verbo búlgaro presenta unas características distintas de las otras lenguas indoeuropeas. La aparición de las formas narrativas, la serie de los perfectos secundarios y otras son innovaciones relacionadas con el valor especial del factor testimonial en la lengua moderna.

PALABRAS CLAVE aoristo, perfecto, testimonialidad, formas narrativas, perfectos secundarios.
RÉSUMÉ Le verbe bulgare presente des characteristiques différentes des autres langues indoeuropéennes. L'apparition des formes de la narration indirecte, la série des parafaits secondaires et d'autres sont des innovations concernant la valeur particulière du facteur testimonial dans la langue moderne.

MOTS CLÉS aorist, parfait, évidence, narration indirecte, parfaits secondaires
ABSTRACT The Bulgarian verb presents many distinctive features compared to other Indoeuropean languages. The appearance of reported forms, series of secondary perfects and others are innovations related to the special signification of the witness factor in the modern language.

KEY WORDS aorist, perfect, witness, reported forms, secondary perfects. 


\section{Introducción}

El verbo búlgaro ha desarrollado un rasgo muy característico relacionado con la testimonialidad del hablante, que opera a distintos niveles: uso del aoristo y otros tiempos pasados, uso del perfecto y formas afines y uso de las formas narrativas.

Este fenómeno no existía en la lengua antigua en la cual, en general, el aoristo era un tiempo de la narración y el perfecto un tiempo del comentario, pero no había una distinción clara y concluyente en el uso de uno $u$ otro. La serie de los perfectos secundarios y las formas narrativas no existían.

\section{La oposición aoristo/perfecto en búlgaro antiguo}

Por el significado, el perfecto no era esencialmente distinto del aoristo, pero a pesar de su proximidad semántica no eran idénticos ya que el aoristo era un pasado absoluto y el perfecto denotaba la relación de la acción anterior con el momento presente.

Sobre la diferencia entre ambos, Vaillant (1948: 346-347) observa que el aoristo, que también tiene un valor de pasado anterior, expone un hecho realizado en el pasado y el perfecto expresa el estado presente que resulta de ese hecho, es decir, presenta una acción pasada desde el punto de vista del presente. La elección entre ambos tiempos a veces es facultativa, y la misma acción puede ser presentada como perteneciente al pasado o trasladada al presente:

Eže oubo Bogŭ sǔčeta (AOR.) člověkŭ da ne razlôčaatǔ (PRES.)

$(M T 19,6)$

Eže ouo Bogŭ sǔčetalŭ estŭ (PERF.) člověkŭ...

[Lo que Dios unió/ha unido, que el hombre no lo separe.]

Comenta Minčeva (1991: 447) que la distribución del perfecto y el aoristo en los manuscritos es muy diversa y depende generalmente del género de los textos, esto no es raro porque los salmos, sermones y oraciones se prestan más al uso del perfecto que los evangelios, que son textos narrativos. La distribución temporal de los originales griegos no se refleja en las traducciones, el perfecto griego se traduce frecuentemente por el aoristo búlgaro. Los contextos semánticos donde se observa la neutralización del perfecto y el aoristo son diversos y frecuentes lo que presupone una proximidad semántica más acusada que ahora y la probabilidad de que en la lengua hablada estuviera mucho más extendido el uso del perfecto. 


\section{La oposición aoristo/perfecto en la lengua moderna}

Desde el punto de vista morfológico el aoristo es un tiempo verbal simple y el perfecto es un tiempo compuesto formado con el presente del verbo auxiliar săm (ser) y el participio pasado acabado (sobre el tema de aoristo) del verbo que se conjuga.

Una misma oración con el verbo en perfecto o en aoristo no se diferencia básicamente por el tiempo objetivo o por el resultado, sino por la manera como la conciencia del hablante toma los factores correspondientes. La diferencia entre el aoristo y el perfecto se basa en que el tiempo de la acción y el del hablante se presenten como diferentes o sean presentados como continuos, respectivamente. El aoristo es un pasado absoluto, el perfecto significa la introducción de un aconecimiento pasado en la esfera del presente:

Levski svi (AOR.) ustni i ne otgovori (AOR.)

[Levski frunció los labios y no respondió.]

V Evropa ne e imalo (PERF.) po-žestoka i po-tragična istorija ot tazi na săvremenna Makedonija.

[En Europa no ha habido una historia más cruel y más trágica que la de la Macedonia contemporánea.]

Hay que remarcar que el perfeco búlgaro no tiene el valor de pasado cercano o inmediato y no se utiliza como tal. El uso del aoristo con carácter de pasado próximo ya aparece documentado en la lengua antigua:

Onǔ že reče (AOR.) emou, bratŭ tvoi pride (AOR.)

$(L C 15,27)$

[Él le dijo: tu hermano ha vuelto.]

Y se mantiene en la lengua moderna:

Vsičko hubavo, lošoto e (PRES.), če zagubihme (AOR.) vojnata, a težko na pobedenija!

[Muy bien, el mal es que hemos perdido la guerra $y_{i}$ ay de los vencidos!]

A lo largo de la evolución de la lengua el perfecto ha adquirido valores diversos: se caracteriza por su valor resultativo, hace referencia a una acción que tuvo lugar en el pasado en un momento indeterminado (aunque se puede explicitar, si conviene, mediante un adjunto) cuyo resultado aún es patente en el momento del discurso. Es desde esta perspectiva que el perfecto se puede considerar un tiempo de la esfera del presente y responde a la formulación " $E$ ant $R$ simul S", un tiempo relativo. 
Por el valor resultativo se justifica el valor inferencial del perfecto: por la situación actual observada se puede inferir una acción pasada que es responsable de esa situación. Puede tratarse de una evidencia lógica, como en este ejemplo:

Njakoj e otvarjal (PERF.) škafa.

[Alguien ha abierto el armario (porque encuentro a la vista un objeto que tenía guardado en el armario).]

Puede tratarse también de una suposición razonable:

Brat mu e (PRES.) mnogo bolen i sigurno napravo pri nego e otišăl (PERF.). [Su hermano está muy enfermo y seguramente ha ido directamente con él.]

El valor resultativo del perfecto con verbos abstractos es más débil, y también puede denotar un resultado no tangible:

Nie sme se vărnali (PERF.) trideset godini nazad.

[Hemos vuelto treinta años atrás.]

Por su indeterminación el perfecto se diferencia del aoristo porque la acción es abstracta en sí misma y se ve desde la perspectiva de su cumplimiento, es decir, del resultado, pero el momento concreto de la acción y el desarrollo particular del proceso son irrelevantes en general. Andrejčin (1977: 240) señala que por esta razón en la gramática tradicional búlgara el perfecto se denomina "pasado indeterminado".

Esta terminología tradicional, sin embargo, no tiene el consenso unánime de todos los autores. Petkov (1987: 44-45), por ejemplo, considera que el término "pasado indeterminado" no es nada adecuado porque a nivel del sistema el perfecto no se diferencia de los otros pasados por el rasgo de indeterminación. Es cierto que a nivel de uso ocupa un lugar especial porque en su significado básico (acción anterior a un momento de orientación presente) está en oposición con el aoristo en su significado básico (acción en un momento determinado anterior al momento del discurso), pero aunque el uso introduzca esta especificidad secundaria del rasgo de indeterminación, no hay base para considerar el perfecto un pasado indeterminado en oposición a los otros pasados. Por otra parte, el propio término "pasado" no tiene el mismo valor en el perfecto ya que hace referencia a un momento de orientación que no es anterior al momento del discurso, sino simultáneo.

En el aoristo actual el carácter testimonial es decisivo y éste es un valor nuevo que en la lengua antigua no existía, cuando la oposición entre aoristo y perfecto no era tan clara. La relación testimonial más especial con la acción que tiene el aoristo actual lo opone a la neutralidad o no-testimonialidad que caracteriza al perfecto (Maslov, 1981: 200-201). 
El rasgo testimonial u "observación directa" en la lengua moderna está íntimamente relacionado con la temporalidad. Son formas testimoniales las del plano del pasado, es decir, las que responden a una fomulación "E rel R ant S", tales como el imperfecto, pluscuamperfecto y futuro pasado, además del aoristo, y son formas neutras las del plano del no-pasado, o sea, las que se formulan "E rel R no-ant S", como el presente, perfecto, futuro y futuro perfecto.

El perfecto moderno es una actualización del aoristo: "E ant S" $\rightarrow$ "E ant R simul $S$ ", y esta actualización permite referirse a hechos pasados de los que el hablante no es testimonio directo.

El desarrollo de las formas narrativas a partir del perfecto (cf. apartado 4) es una muestra del carácter no-testimonial que los hablantes le atribuyeron en oposición a la testimonialidad asociada al aoristo.

\section{Las formas narrativas}

El búlgaro moderno presenta un rasgo característico que no tiene paralelo en otras lenguas indoeuropeas: cuando el hablante está refiriendo hechos anteriores que no ha visto ni ha deducido personalmente, sino que conoce a través del relato de otra persona (artículos, libros, documentos antiguos, películas, conversaciones, etc.), no puede utilizar los tiempos pasados testimoniales y se ha de servir de las llamadas formas narrativas. Morfológicamente son formas compuestas sobre el modelo del perfecto (que coincide con el aoristo narrativo) que incluyen también formas con el participio pasado inacabado (sobre el tema de imperfecto). Es característica de las formas narrativas la pérdida del auxiliar en las terceras personas:

Blagorodna i bogata, slavna i blagočestiva bila (IMPERF. NARR.) hristianskata familija, v kojato toj se rodil (AOR. NARR.) i izrasnal (AOR. NARR.).

[Noble y rica, gloriosa $y$ honorable era la familia cristiana en la cual él nació $y$ creció.]

Las formas narrativas de hecho son, o pueden ser, semánticamente coincidentes con el discurso indirecto, pero así como en éste es la sintaxis oracional la que indica la fuente indirecta, con las formas narrativas es la propia morfología verbal la que lo indica.Véase la diferencia entre estos dos ejemplos:

Radoslavov sam beše potvărdil (PLUSCUAM.), če toj dărži (PRES.) komiteta v răcete si.

[Radoslavov mismo había afirmado que él tomaba el comité en sus manos.] 
Tezi uspehi na bălgarite se ulesnjavali (IMPERF. NARR.) otčasti ot anarhijata, kojato gospodsvuvala (IMPERF. NARR.) văv Vizantija.

[Estos éxitos de los búlgaros se beneficiaban en parte de la anarquía que reinaba en Bizancio.]

El origen de las formas narrativas no está muy claro. Parece ser que empezaron a utilizarse durante la ocupación otomana, cuando capas de la población eran más o menos bilingües, porque en turco existe un pasado para referirse a hechos no presenciados personalmente formado con un verbo auxiliar y un participio, formalmente semejante al perfecto búlgaro.

Otra posibilidad de explicación sería la reducción sintáctica como señala Ivančev (1976: 355-356): el discurso indirecto se expresa mediante una oración subordinada completiva dependiente de un verbo principal de habla y la testimonialidad del aoristo obliga al uso del perfecto en la subordinada:

Toj kazva (PRES.), če Ivan e čel (PERF.) knigata.

[Él dice que Iván leyó/ha leído el libro.]

En esa situación concreta el verbo auxiliar en tercera persona podría omitirse, y de ahí se pasó a la omisión de la propia oración principal, que de hecho no aportaba nada al acto comunicativo puesto que la forma verbal por sí misma ya aportaba el valor de conocimiento indirecto:

Ivan čel (AOR. NARR.) knigata.

[(Alguien dice que) Iván leyó el libro.]

He aquí un ejemplo del uso del aoristo narrativo en el discurso indirecto que sugiere ese origen:

Edin star pop mi kazvaše (IMPERF.), koga došel (AOR. NARR.) p'rvijt g'rski vladika văv Voden, sobral (AOR. NARR.) site bugarski knigi ot c'rkvite i ne znajat (PRES.) što im činil (AOR. NARR.).

[Un anciano sacerdote me contaba (que) cuando llegó el primer obispo griego a Edesa reunió todos los libros búlgaros de las iglesias y no saben quéles hizo.]

En la oración subordinada el uso de las formas narrativas no es obligado puesto que la sintaxis oracional ya explicita el discurso indirecto, pero no es nada infrecuente encontrar formas narrativas en este tipo de oraciones: 
V čaršijata njakoj mi kaza (AOR.), če Marin otišăl (AOR. NARR.) s părvencite v konaka.

[En el mercado alguien me dijo que Marin fue con los notables a la alcaldía.]

V taka narečenata Solunska legenda se kazva (PRES.), če na solunskoto tărženie se govorelo (IMPERF. NARR.) i na bălgarskija ezik.

[En la llamada leyenda de Salónica se dice que en el mercado de Salónica se hablaba también en búlgaro.]

Por el carácter testimonial de las formas del plano del pasado es lógico que sea obligatorio el uso de las formas narrativas cuando nos referimos a acontecimientos del pasado que conocemos sólo indirectamente:

Spored drua versija Protogerov nikoga ne bil kazval (PLUSCUAM. NARR.) na carja, če priema (PRES.) plana za prevrat, i bil otklonil (PLUSCUAM. NARR.) predloženieto.

[Según otra versión, Protogerov nunca había dicho al zar que aceptaba el plan de golpe de estado y había rehusado la propuesta.]

En el plano del no-pasado el uso de las formas narrativas sólo es optativo y el hablante suele utilizarlas para distanciarse de aquello que relata, para no hacerse responsable porque no está seguro de que sea cierto, porque está en contra, etc.:

Poradi izvestna fizičeska prilika s car Boris, săštesvuvaha (IMPERF.) sluhove, če Draganov bil (IMPERF. NARR.) sin na car Ferdinand.

[Por el visible parecido físico con el zar Boris, corrían rumores que Draganov era hijo del zar Fernando.]

Hay que señalar que las formas narrativas se caracterizan por la neutralización del rasgo "pasado" y esto presupone que las formas temporales que sólo se diferencian por la relación "R ant S"/"R simul S" tienen un correlato narrativo común, excepto el aoristo que es una forma absoluta que no tiene un momento de referencia porque es el propio aoristo el que lo marca. Así pues, el imperfecto narrativo se corresponde tanto al presente como al imperfecto, etc. La correspondencia entre formas no-narrativas y narrativas es como sigue: 


\begin{tabular}{|r|l|}
\hline FORMAS NO-NARRATIVAS & FORMAS NARRATIVAS \\
\hline $\begin{array}{r}\text { Presente } \\
\text { Imperfecto }\end{array}$ & Imperfecto narrativo \\
\hline $\begin{array}{r}\text { Perfecto } \\
\text { Pluscuamperfecto }\end{array}$ & $\begin{array}{l}\text { Pluscuamperfecto } \\
\text { narrativo }\end{array}$ \\
\hline Aoristo & Aoristo narrativo \\
\hline Futuro & Futuro pasado narrativo \\
\hline Futuro pasado & \\
\hline
\end{tabular}

Durante mucho tiempo las formas narrativas fueron etiquetadas de mjuy diversas maneras en las gramáticas. Teodorov-Balan (1957) fue el primero en sistematizarlas de forma clara y coherente y demostrar que formaban un subsistema con carácter modal que él llamó "modo narrativo", que se ha mantenido en las gramáticas durante años. Andrejčin (1978: 244-245) ya opina qjue las formas narrativas no son una categoría modal autónoma, sino que el modo indicativo tiene dos clases de formas de la narración: las directas y las indirectas o narrativas. Sin embargo, en la Gramática de la lengua literaria búlgara contemporánea (1983) auspiciada por la Academia de ciencias, este mismo autor incluye entre la descripción de los modos la del "modo narrativo" (p. 351).

El modo imperativo y el condicional también pueden tener sus formas narrativas (aunque su uso popular es mucho más exiguo), lo cual comenzó a poner en duda la modalidad narrativa desde la década de los ochenta, aunque diversos autores han seguido hablando del "modo narrativo" por tradición. Lindstedt (1985: 263) no cree que la categoría de modo esté definida tan exactamente que se precise diferenciarla esrictamente de la categoría evidencial; cuando un significado está gramaticalizado se puede etiquetar de modo. Concluye este autor que es razonable asumir que el sisema de las formas narrativas constituye un modo que implica una reserva epistémica del hablante basándose en que transmite información indirecta.

Kucarov (1998: 413-417) niega categóricamente que las formas narrativas pertenezcan a la categoría de modo y las asigna a una categoría mofológica autónoma que de denomina "aspecto del discurso" (vid na izkazvaneto), cuyo contenido semántico se basa en la relación del hablante con el discurso.

\section{Situación de los perfectos secundarios}

En las oraciones subordinadas sustantivas que conforman el discurso indirecto es posible mantener la misma forma temporal de la oración en el discurso directo, sea cual sea la forma del verbo en la principal: 
Ivan kaza (AOR.): šte pristigna (FUT.) skoro.

[Iván dijo: llegaré pronto.]

Ivan kazva (PRES.) / kaza (AOR.) / e kazal (PERF.) / šte kaže (FUT.), če šte pristigne (FUT.) skoro.

[Iván dice/dijo/ha dicho/dirá que llegará pronto.]

Esta particularidad de la concordancia búlgara permite la paradoja de que el perfecto en una oración subordinada pueda proyectarse atrás en el tiempo denotando un pasado más lejano que el pluscuamperfecto en la principal:

Karavelov mu beše săobštil (PLUSCUAM.), če e vljazal (PERF.) văv vrăzka s oražejnata firma Remingtăn.

[Karavelov le había informado que había entrado en contacto con la empresa de armamento Remington.]

Cuando el verbo de la oración principal está en pasado también es posible una solución paralela a las del castellano y catalán:

Ivan kaza (AOR.), če šteše da pristigne (FUT. PAS.) skoro.

[Iván dijo que llegaría pronto.]

Pero existe una restricción en la concordancia, relacionada con el factor testimonial. Cuando el verbo de la subordinada, que representa el discurso directo, debiera estar en aoristo, éste se debe pasar a perfecto, y cuando debiera aparecer en imperfecto, se pasa a perfecto secundario, que es una forma compuesta semejante al perfecto, pero con el participio pasado inacabado, o sea, formado sobre la base del imperfecto. De esta manera se mantiene en el discurso indirecto la oposición aoristo/ imperfecto del discurso directo, que es una oposición tempoaspectual muy antigua:

Hristo kazva (PRES.): ostavih (AOR.) pismoto na bjuroto.

[Hristo dice: dejé la carta sobre la mesa.]

Hristo kazva (PRES.), če e ostavil (PERF.) pismoto na bjuroto

[Hristo dice que dejó / ha dejado la carta sobre la mesa.]

Toj kaza (AOR.): prez ljatoto Plamen četeše (IMPERF.) cjal den.

[Él dijo: en verano Plamen leía todo el día.]

Toj kaza (AOR.), če prez ljatoto Plamen e četjal (PERF. SECUND.) cjal den.

[Él dijo que en verano Plamen leía todo el día.] 
Hay que notar que en los verbos de la III conjugación (vocal temática -a-) no hay diferencia formal entre ambos perfectos porque los dos participios son idénticos $y$, por otra parte, en algunas hablas occidentales el participio sobre la base del imperfecto no existe y el perfecto cubre también las funciones de su homónimo secundario.

El perfecto es una actualización del aoristo según se ha dicho, y el perfecto secundario es una actualización del imperfecto, que presenta una formalización más compleja: "E simul R ant S" $\rightarrow$ "E simul R ant R' simul S". Otro tanto se puede decir del pluscuamperfecto y del futuro pasado secundarios, que son actualizaciones de sus homónimos primarios. La gramática tradicional ha prestado poca atención a estas formas y de aquí que, según Petkov (1987: 38), no se haya entablado un debate científico en profundidad sobre la naturaleza, semántica y posición en el paradigma de los perfectos secundarios y por esta razón se mantienen opiniones muy diversas.

Andrejčin (1978: 227-234) las considera formas narrativas que conservan el auxiliar en tercera persona, lo cual resulta dudoso cuando menos, puesto que una característica de las formas narrativas es la omisión del auxiliar en tercera persona. Kucarov (1994: 5) ve en estas formas la expresión de un modo conclusivo o deductivo. Penčev (1987: 471), en cambio, afirma que son realmente formas de perfecto y no hay que basarlas en el carácter inferencial porque en muchos casos no reflejan ninguna idea de suposición o deducción. Aunque sean morfológicamente idénticas, las formas de la serie de los perfectos secundarios son esencialmente distintas del modo conclusivo descrito por Kucarov (1994).

Lindstedt (1985: 267-268) justifica la situación de los perfectos secundarios dentro del sistema con el hecho que elperfecto tiene un doble estatus: por una parte se opone a las otras formas de indicativo por sus referencias temporales, por otra parte sirve como contrapartida evidencialmente neutra, a veces inferencial, del aoristo. Pero las otras formas testimoniales de indicativo, es decir, los tiempos del plano del pasado, en principio pueden tener también su contrapartida neutra, puesto que no todos los hechos no presenciados personalmente por el hablante han de ser conocidos necesariamente a través de otra persona, que sería el caso de las formas narrativas. Los perfectos secundarios forman parte del subsistema de las formas de pasado evidencialmente neutras, opuestas a las formas testimoniales por una parte y a las formas narrativas por otra.

\begin{tabular}{|c|c|c|}
\hline FORMAS TESTIMONIALES & FORMAS NEUTRAS & FORMAS NARRATIVAS \\
\hline Aoristo & Perfecto & Aoristo narrativo \\
\hline Imperfecto & Perfecto secundario & Imperfecto narrativo \\
\hline Pluscuamperfecto & Pluscuamperfecto secundario & Pluscuamperfecto narrativo \\
\hline Futuro pasado & Futuro pasado secundario & Futuro pasado narrativo \\
\hline
\end{tabular}




\section{Conclusiones}

Sería interesante averiguar en qué momento histórico aparecieron las innovaciones aquí descritas y se instalaron en la lengua de forma sistemática, pero esto no es posible a causa de la fractura cultural que se operó durante la larga ocupación otomana (1396-1878), en la época justamente en que se gestaba la lengua moderna. La cultura quedó limitada a los monasterios y la escasa producción literaria, generalmente de carácter religioso, siguió utilizando por inercia la lengua antigua, esclerosada en una morfosintaxis que ya no respondía al uso de los hablantes.

Tenemos testimonios de la lengua viva real en algunos textos del siglo XVIII y sobre todo a partir de principios del siglo XIX, cuando ya habían cristalizado todos los grandes cambios dentro del sistema y las peculiares innovaciones en el campo de la morfología verbal.

En las gramáticas antiguas y no tan antiguas se observan muchas vacilaciones, omisiones e incluso contradicciones para situar dentro del paradigma las formas innovadas y la frecuencia de las homonimias morfológicas dificultó sin duda el estudio sistemático de las mismas. Tampoco hallamos referencias explícitas al carácter exclusivamente testimonial del aoristo y los otros tiempos del plano del pasado, aunque algunos autores describían el aoristo como un "pasado determinado", frente al perfecto, presentado como un "pasado indeterminado", pero la terminología en éste y otros aspectos es poco precisa en general.

Puede hallarse una descripción minuciosa sobre la evolución de los conceptos en la gramática búlgara, por lo que respecta al verbo, en Părvev (1975) y los criterios aceptados actualmente con notable unanimidad en Kucarov (1998).

La aparición de los nuevos fenómenos que afectaron al sistema verbal está relacionada quizá con la evolución de la lengua viva hacia el analitismo y con el hecho de que en casi todas las lenguas eslavas el perfecto amplía en gran medida su campo semántico y llega a suplantar a los pasados simples (aoristo e imperfecto) que llegan a desaparecer, excepto en el conjunto de las hablas búlgaro-macedonias, donde se conservan bien vivos. Esta coexistencia tardía bien pudo llevar hacia una reubicación y nueva especialización semántica de las formas verbales disponibles. 


\section{REFERENCIAS}

ANDREJČIN, L. (1977) “Glagol”, en Andrejčin L., K. Popov \& S. Stojanov, Gramatika na bălgarskija ezik, Sofija, Nauka i izkustvo, pp. 190-296.

ANDREJČIN, L. (1978) Osnovna bălgarska gramatika, Sofia, Nauka i izkustvo.

ANDREJČIN, L. (1983) “Naklonenie na glagola”, en Stojanov S., K. Ivanova, P. Pašov \& V. Stankov (eds.) Gramatika na săvremennija bălarski knižoven ezik, vol. 2: Morfologija, Sofija, BAN, pp. 351-372.

IVANČEV, SV. (1976) "Problemi na razvitieto i funkcioniraneto na modalnite kategorii v bălgarskija ezik", en Pašov A \& R. Nicolova (eds.), Pomagalo po bălgarska morfologija. Glagol, Sofija, Nauka i izkustvo, pp. 348-359.

KUCAROV, IV. (1994) Edno ekzotično naklonenie na bălgarskija glagol, Sofija, Sofijski universitet Sv. Kliment Ohridski.

KUCAROV, IV. (1998) “Morfologija”, en Bojadžiev T., Iv. Kucarov y J. Penčev, Săvremenen bălgarski ezik, Sofija, Petăr Beron, pp. 277-497.

LINDSTEDT, J. (1985) On the Semantics on Tense and Aspect in Bulgarian, Slavica Helsingiensia 4, Helsinki, University of Helsinki.

MASLOV, Ju.S.(1981) Grammatika bolgarskogo jazyka, Moskva, Vysšaja Škola (Gramatika na bălgarskija ezik, Sofija, Nauka i izkustvo, 1982).

MINČEVA, A. (1991) “Upotreba na vremena i naklonenija v izrečenieto”, en Duridanov IV., E. Dogramadžieva \& A. Minčeva (eds.) Gramatika na starobălgarskija ezik, Sofija, BAN, pp. 444-449.

PĂRVEV, Hr. (1975) Očerk po istorija na bălgarskata gramatika, Sofija, Nauka i izkustvo.

PENČEV, J. (1987) “Perfekt i prevrăštane v perfekt”, Dokladi ot II Meždunaroden kongres po bălgaristika, vol. 3, pp. 468-474.

PETKOV, P. (1987) “Kăm văprosa za săstava na temporalnata paradigma v bălgarskija ezik”, Săpostavitelno ezikoznanie, vol. XII n² ${ }^{\circ}$, pp. 37-49.

TEODOROV-BALAN Al. (1957) “Četvărto naklonenie”, Bălgarski ezik, 4: 305-317.

VAILLANT A. (1948) Manuel du vieux slave, vol. 1: Grammaire, Collection de manuels de l'IES, VI. Paris, Institut d'Études Slaves, ed. 1964. 